\title{
The attitude of medical students towards the mental mentally ill: the impact of a clinical attachment in Psychiatry
}

\author{
Angelo de Alwis, Rasitha Perera, HL Vasantha, Thushani Henegama, Sunera Fernando
}

\section{Background}

Working and associating with people with mental illness influence the attitudes towards mental illness. Medical students of the Faculty of Medicine, University of Colombo, Sri Lanka have a mandatory six week clinical attachment in psychiatry in the final year, which provides an opportunity to work with people with mental illness.

\section{Aims}

To determine the impact of the clinical attachment in psychiatry on attitudes among medical students towards people with mental illness.

\section{Method}

The 20-point Attitude to Mental Illness Questionnaire (AMIQ) was used to assess stigmatizing attitudes, before and after the six week clinical attachment in psychiatry, in a cohort of medical students completing the appointment during a one year period January-December 2011. The AMIQ consists of five hypothetical case vignettes and five questions based on each case vignette.

\section{Results}

Questionnaires were received from 194 medical students, $(88 \%$ response rate) before the commencement of the clinical attachment and from 137 medical students, (62\% response rate) after completing the clinical attachment. The post attachment mean AMIQ stigma score had improved for schizophrenia, depression, heroin use and alcohol use. The difference in AMIQ stigma scores were significant for heroin use $(p=0.014$, median difference $=0.4)$, schizophrenia $(p=0.049$, median difference $=0.3$, and depression $(p=0.014$, median difference $=0.4)$ In both the pre-clinical attachment as well as the post-clinical attachment groups, stigmatizing attitudes were significantly higher for depression $(p<0.0001)$, and schizophrenia $(p<0.001)$ compared to the attitude towards diabetes.

Conclusion

The final year clinical attachment in psychiatry significantly reduced stigmatizing attitudes among medical students towards mental illness.

SL J Psychiatry 2012; 3 (2):12-15

\section{Introduction}

Attitudes of medical students towards people with mental illness are influenced by many factors. Training in psychiatry and experiences in medical school play an important role in determining these attitudes (1). Therefore, a clinical rotation in psychiatry is an opportunity to influence the attitudes of medical students towards psychiatry as a discipline as well as their attitude towards those with mental illness.

Medical student have negative attitudes towards mental illnesses and the discipline of psychiatry (2). These attitudes have an effect on the quality of service delivery as well as in their future choice of specialization in psychiatry (3). The negative attitude toward psychiatry stems from misconceptions students have and thus could be corrected during the psychiatry clerkship (4).

Direct contact with patients with mental illness can reduce or sometimes increase stigmatizing attitudes (5-9). Positive educational experiences and positive personal experiences have been shown to improve attitudes (5-8). Other research has shown that being exposed to persons with long term mental illness as well as being exposed to negative images of mentally ill people may worsen attitudes (9).
Compared to the main four clinical disciplines of medicine, surgery, gynecology and obstetrics and paediatrics, medical students have exposure to psychiatry. The Faculty of Medicine in the University of Colombo is the first medical school in Sri Lanka to introduce a psychiatry rotation as part of the clinical training in the final year. At present, final year clinical attachments in psychiatry are conducted in several other medical faculties in the country. During these rotations medical students have the opportunity to have direct contact with people with mental illnesses.

Several studies have looked at the influence on attitudes of different clinical programmes and varying durations of clerkship on attitudes (10-11). Researchers at the University of Kelaniya, Sri Lanka demonstrated favorable improvements in the ATP-30 score following exposure to the professorial clerkship in psychiatry in medical undergraduates as well as significant increase in the desire to pursue a career in psychiatry among medical undergraduates following exposure to the professorial clerkship in psychiatry (12).

\section{Methods}

We recruited a cohort of 194 medical students, undertaking the final year psychiatry rotation in the 
Faculty of Medicine, University of Colombo. This cohort of medical students completed their mandatory psychiatry rotation in five consecutive groups, in the University Psychiatry Unit at the National Hospital, Colombo, Sri Lanka from January to December 2011.

\section{Clinical attachment}

The University Psychiatry Unit of the Faculty of Medicine, Colombo is a General Hospital psychiatry unit, which provides in-patient care to approximately 1000 mentally ill individuals annually. It also provides Consultation Liaison services to the National Hospital and out-patient care. During the clinical attachment medical students have the opportunity to clerk inpatients and out-patients with psychiatric illness, and participate in rehabilitation and community based care. They have an opportunity to visit community based organizations and a child psychiatry clinic at a teaching hospital. Students also participate in small group teaching sessions each day. The clinical attachment ends with a clinical assessment, where the students are tested on their ability to assess and manage patients with psychiatric illness.

The investigators did not directly influence the content of any teaching sessions or carry out special teaching on stigma in mental illnesses.

\section{Outcome measures}

The Attitude to Mental Illness Questionnaire (AMIQ) was administered on the first day of the psychiatry rotation. The questionnaire consisted of five short vignettes describing hypothetical patients and five questions based on each of the case vignettes. The short case vignettes described people diagnosed with schizophrenia, depression, alcohol dependence, heroin dependence and diabetes. The case vignettes included the following.

- Janaka has been injecting heroin for the last 1 year.

- Thilina is depressed. He took a overdose of paracetamol last month to tried to hurt himself.

- Manjula has schizophrenia. He needs medication in injectable form every two weeks. He was detained in hospital for several weeks two years ago because he was hearing voices from the devil and thought that he had the power to cause earth quakes. He had been detained under the Mental Health Act in the past.

- $\quad$ Piyal has diabetes. He needs to inject insulin everyday and is on a special diet.

- Sirisena has been drinking heavily for five years. He now attends Alcoholics Anonymous meetings.
The questions on each case vignette explored whether the respondent thought the illness would damage the patient's career, if they were comfortable having the patient as a colleague or socializing with him, whether they thought it was likely that the patient's wife would leave him, or whether the patient will encounter difficulties with the law. The questionnaire given in the pre-clinical phase, contained four additional questions which inquired about family member or friends with psychiatric illness.

The students answered the same questionnaire at the end of the six week clinical attachment. Questionnaires were coded to identify the pre and post test responses of individual students.

Responses to individual questions were scored on a five point Likert Scale (maximum +2 , minimum -2) with unanswered questions, 'neutral' and 'don't know' answers scoring zero.

\section{Statistical analysis}

Data was analyzed using version 17 of the SPSS statistical programme. Due to the small sample size and the non-normal distribution of the data, the nonparametric test Wilcoxon signed-rank test was used to compare the pre rotation AMIQ scores with the post rotation AMIQ score. The Mann-Whitney U test was used to investigate associations between stigma scores and factors which influence attitudes.

\section{Results}

Completed questionnaires were returned by 194 medical students, ( $88 \%$ response rate) before the commencement of the clinical attachment in psychiatry and 137 medical students, (62\% response rate) after completing the clinical attachment. The mean age of our sample was 25.2 years $(\mathrm{SD}=0.84)$. The sample consisted of 87 female medical students (47\%).

Only 88 pairs of questionnaires could be identified to perform the Wilcoxon signed-rank test for change in stigma scores between the pre and post psychiatry rotation groups. This was because some medical students had incorrectly coded the post psychiatry rotation questionnaire.

The mean AMIQ scores for schizophrenia, depression, alcohol use and heroin use before and after the psychiatry rotation are given in table 1 . The mean

\begin{tabular}{|l|l|l|l|}
\hline \multicolumn{4}{|c|}{ Table 1-AMIQ scores before and after the psychiatry } \\
rotation
\end{tabular}

\begin{tabular}{|l|l|l|l|l|}
\multicolumn{5}{|c|}{ Table 2 - Sigma scores for different types of diagnoses } \\
& Schizophrenia & Depression & Alcohol & Heroin \\
\hline Friends & 0.948 & 0.155 & 0.904 & 0.523 \\
Family & 0.572 & 0.099 & 0.472 & 0.258 \\
$\begin{array}{l}\text { Future } \\
\text { plans } \\
\text { Difficulty }\end{array}$ & 0.165 & 0.465 & 0.973 & 0.517 \\
\hline
\end{tabular}


AMIQ score had improved for schizophrenia, depression, heroin use and alcohol use, following the completion of the psychiatry rotation. The difference in AMIQ stigma scores were significant for heroin use $(p=0.014$, median difference $=0.4)$, schizophrenia $(p=0.049$, median difference $=0.3)$, and depression $(\mathrm{p}=0.014$, median difference $=0.4)$. However, there was no significant improvement in the score for alcohol use $(p=0.068$, median difference $=0.4)$.

Seventy four participants said they knew a friend with mental illness and 18 said they had a family member with mental illness. When asked about their willingness to pursue psychiatry as a career, 20 (10.4\%) said they had such plans. The answers given to these two questions were not significantly associated with the stigma scores of the various mental illnesses (Table 2).

\section{Discussion}

This study suggests that the attitude towards schizophrenia, depression and heroin use improved following a clinical attachment in psychiatry. In addition the pre-psychiatry clinical attachment stigma scores demonstrate that students have a more stigmatizing attitude towards the mental illnesses schizophrenia, depression, alcohol and heroin abuse compared to the physical illnesses diabetes. This is consistent with findings of other studies (2).

In other studies with a similar design, investigators have shown improvement in the attitudes of medical students following a psychiatry rotation of six weeks or more $(7,8,10)$. However some studies have not shown improvement of the stigma scores after a psychiatry clinical attachment especially when the duration of the clinical rotation is two weeks or less $(9,11)$. Thus the duration of the clinical rotation seems to be an important factor influencing attitudes. Other factors that contribute to a demonstrable improvement in attitudes are medical students taking individually assigned patients and clerking them, emphasis on the doctor-patient relationship and being exposed to mental illnesses that respond quickly to treatment (13-14). In this clinical attachment, all these factors were fulfilled. In addition to these, the students were also exposed to community psychiatry, community based organizations and consumer and carer groups. These may have contributed towards improving stigma scores.

Stigma score improvement in our sample was seen only for schizophrenia, depression and heroin use. Stigma scores did not improve for alcohol use. Being exposed less to individuals with alcohol use during the clinical rotation may have contributed to this.

The limitations of the study are the small sample size and the high attrition rate. In addition the students may also have been influenced by the perceived need to 'please' their teachers at the end of psychiatry rotation, which could have improved the stigma scores.

This study demonstrates that the rotation in psychiatry is a valuable opportunity to influence attitudes of medical students. Further research is needed to identify the specific factors that contribute to positive change in attitudes. Comparison of attitudes among medical students in the different universities may help identify some of these factors.

\section{Declaration of interest}

None declared.

\author{
Angelo de Alwis, Registrar in Psychiatry \\ Rasitha Perera, Registrar in Psychiatry \\ HL Vasantha, Registrar in Psychiatry \\ Thushani Henegama, Registrar in Psychiatry \\ University Psychiatry Unit, NHSL, Colombo, \\ Sri Lanka \\ Sunera Fernando, Lecturer \\ Department of Psychological Medicine, Faculty of \\ Medicine, University of Colombo, Sri Lanka \\ Corresponding author \\ Angelo de Alwis \\ E mail: angelo.dealwis@gmail.com
}

\section{References}

1. Fischel T, Manna H, Krivoy A, Lewis M, Weizman .: Does a Clerkship in Psychiatry Contribute to Changing Medical Students' Attitudes Towards Psychiatry? Acad Psychiatry 2008; 32:147-150 CrossRef

2. Fernando SM, Deane FP, McLeod H.: Sri Lankan doctors' and medical undergraduates' attitudes towards mental illness. Soc Psychiatry Psychiatr Epidemiol. 2010; 45(7):733-9 CrossRef

3. Nielsen, AC, Eaton, J. Medical students' attitude about psychiatry. Implications for psychiatric recruitment. Arch Gen Psychiatry 1981; 38:1144-1154 CrossRef

4. Feifel D, Moutier CY, Swerdlow NR. Attitudes toward psychiatry as a prospective career among students entering medical school. Am J Psychiatry 1999; 156:1397-1402 PMid:10484951

5. Alexander, DA Eagles, JM. Changes in attitudes towards psychiatry among medical students: correlation of attitude shift with academic performance. Med Educ1990; 24 (5): 452-460 $\underline{\text { CrossRef }}$

6. Shelley, RK., Webb, MG. Does clinical clerkship alter students' attitudes to a career choice of psychiatry. Med Educ, 1986; 20(4): 330-334 CrossRef

7. Chung KF: Changing the attitudes of Hong Kong medical students toward people with mental illness. J Nerv Ment Dis. 2005; 193(11):766-8 CrossRef

8. Coodin S, Chisholm F. Teaching in a new key: effects of a co-taught seminar on medical students' attitudes toward schizophrenia. Psychiatr Rehabil J. 2001; 24(3): 299-302 PMid:11315216

9. Rezler AG; Attitude changes during medical school: a review of the literature. J Med Educ. 1974; 49 (11):1023-30 PMid:4613834

10. Rajagopalan M, Kuruvilla K. Medical students' attitudes to psychiatry: the effect to of a four week posting. Indian Journal of Social Psychiatry 1987; 3(3):238-259

11. Rajagopalan, M, Kuruvilla, K; Medical students' attitudes towards Psychiatry: effect of a two week posting; Indian J. Psychiat 1994: 36 (4): 177-182 PMid:21743698 PMCid:2972500 
de Alwis, Perera, Vasantha, Henegama and Fernando

12. Rodrigo, A, Wijesinghe, C, Kuruppuarachchi KALA. Changes in attitudes toward psychiatry with introduction of a new curriculum: experiences of a Sri Lankan medical school. SL J Psychiatry 2012; 3 (1):14-16 CrossRef

13. Lau AYH, Offord DR. A study of student attitudes towards a psychiatric clerkship 1976: J Med Educ 1976; 51 (11) 919-928 PMid:978703

14. Eagle, PF, Marcos, LR. Factors in medical students' choice of psychiatry. Am J Psychiatry 1980; 137(4): 423-427 PMid:7361926 